\title{
Retrospective Study as First-Line Chemotherapy Combined Anti-VEGF Antibody with Fluoropyrimidine for Frail Patients with Unresectable or Metastatic Colorectal Cancer
}

\author{
Motoki Yoshida Masahiro Goto Takayuki Kii Hitoshi Nishitani \\ Shinichiro Kawabe Shin Kuwakado Ken Asaishi Takahiro Miyamoto \\ Kazuhide Higuchi
}

Division of Cancer Chemotherapy Center and Gastroenterology, Osaka Medical College Hospital, Osaka, Japan

\author{
Key Words \\ Bevacizumab - Colorectal cancer • First-line chemotherapy • \\ Fluoropyrimidine $\cdot$ Frail patients $\cdot \mathrm{S}-1$
}

\begin{abstract}
Background/Aim: Combination chemotherapies of oxaliplatin or irinotecan with fluoropyrimidine and molecular target drug were reported to be active in several clinical studies and so regarded as a first-line standard therapy for unresectable or metastatic colorectal cancer. However, the incidence of adverse events is not so low. We investigated the efficacy and safety of chemotherapy combined bevacizumab with fluoropyrimidine as a first-line treatment for frail patients. Methods: Twenty-six patients with unresectable or metastatic colorectal cancer who were treated with first-line chemotherapy combined bevacizumab with S-1 or 5FU/LV (modified Roswell Park Memorial Institute regimen) at our hospital between October 2007 and December 2010 were retrospectively investigated. Results: The median age was 72 years (range 66-84). Performance status was 0,1 and 2 in 8,17 and 1 patient, respectively. The primary lesion was located in the colon in 14 patients and in the rectum in 12 .
\end{abstract}

Twenty patients were with resection of the primary lesion and 6 were without, 8 were with postoperative adjuvant chemotherapy and 18 were without. The number of metastasized organs was 1, 2 and 3 in 17, 9 and 0 patients, respectively. The liver, lung, lymph node and peritoneum were metastasized in 9, 9, 11 and 5 patients, respectively. The KRAS gene was wild in 11, mutated in 7 and unknown in 8 patients. Bevacizumab with S-1 was used in 17 patients and bevacizumab with 5FU/LV was used in 9. Response and disease control rates were 50 and $100 \%$, respectively. The median duration of progression-free survival was 9.1 months and the median time to treatment failure was 9.0 months. The incidences of all grades of neutropenia and hypertension were $31 \%$, those of grade 3 or severer were $12 \%$, and those of other adverse events were low. Grade 3 cerebral hemorrhage, grade 4 pulmonary embolism and grade 5 febrile neutropenia each occurred in 1 patient. Conclusion: The first-line chemotherapy combined bevacizumab with fluoropyrimidine for frail patients with unresectable or metastatic colorectal cancer in Japan was comparable to the safety and efficacy of combination therapy reported previously in Western countries.

Copyright $\odot 2013$ S. Karger AG, Basel

\section{KARGER}

Fax +4161306 1234

E-Mail karger@karger.ch

www.karger.com (c) 2013 S. Karger AG, Basel

$0012-2823 / 13 / 0871-0059 \$ 38.00 / 0$

Accessible online at:

www.karger.com/dig
Motoki Yoshida

Division of Cancer Chemotherapy Center and Gastroenterology

Osaka Medical College Hospital, 2-7 Daigakumachi, Takatsuki

Osaka 569-8686 (Japan)

E-Mail ctc004@poh.osaka-med.ac.jp 


\section{Background}

The median overall survival (OS) was about 2 years in several pivotal phase III studies of chemotherapy for unresectable or metastatic colorectal cancer, in which combination therapy with 3 anticancer drugs (oxaliplatin or irinotecan and fluoropyrimidine) and a molecular target drug administered as a first-line treatment showed efficacy, and these therapies are considered as the standard treatment [1-3].

In several phase III clinical studies performed before the introduction of any molecular target drug, oxaliplatin or irinotecan and fluoropyrimidine combination chemotherapy as a first-line treatment were compared with the sequential chemotherapy started with fluoropyrimidine treatment followed by oxaliplatin or irinotecan as the second-line treatment, and no significant difference was noted in the median OS in any of these clinical studies [4-6]. The response rate was higher in combination chemotherapy, whereas progression-free survival (PFS) of sequential chemotherapy was comparable. Regarding adverse events, toxicity was lower in sequential than in combination chemotherapy, attracting attention.

It has been found that cure or long-term survival may be achieved when curative resection of the liver is performed after a response to chemotherapy in patients with liver metastasis alone [7]. As a therapeutic strategy, it has been proposed to select combination chemotherapy for patients requiring an active approach in whom curative resection may be possible after responding to chemotherapy, and sequential chemotherapy for patients for whom stability of the disease condition is important rather than responses and also those without a tumorassociated symptom not requiring an aggressive approach [8].

On the other hand, many colorectal cancer patients are elderly, aged 65 years or older, in whom the incidence of complications is high and many are frail patients. The incidence of adverse events, particularly neutropenia, is high in combination chemotherapy in the elderly compared to that in the non-elderly, although the efficacy is similar [9-14]. The characteristics of adverse events induced by 5 types of anticancer drug effective for colorectal cancer are as follows: the incidence of anti-epidermal growth factor receptor (EGFR) antibody preparation-induced skin toxicity is high, oxaliplatin is likely to protract peripheral sensory neuropathy, irinotecan induces diarrhea, malaise and depilation, bone marrow toxicity of oxaliplatin and irinotecan is slightly high, whereas the incidences of gastrointestinal hemorrhage/perforation and thrombosis caused by an anti-vascular endothelial growth factor (VEGF) antibody preparation, bevacizumab, are very low, and the incidence of severe adverse events induced by fluoropyrimidine is lower than those induced by oxaliplatin and irinotecan. These characteristics suggest that the incidence of adverse events in chemotherapy combined bevacizumab with fluoropyrimidine may be lower than that in 3-drug combination therapy, making it more suitable as the first-line chemotherapy for frail patients, mainly the elderly.

The usefulness of chemotherapies combined bevacizumab with 5FU/LV or capecitabine has been shown in several clinical studies: these were administered to frail patients as a first-line treatment in the AVF2192g study [15] and as a sequential chemotherapy in the MAX study [16]. The median PFS times achieved by chemotherapy combined bevacizumab with fluoropyrimidine in these studies were 9.2 and 8.5 months, respectively, which were comparable to those achieved in pivotal phase III studies in which oxaliplatin or irinotecan was additionally combined (9.4 [1], 8.9 [2] and 9.6 months [3]).

In Japan, chemotherapy combined bevacizumab with S-1 achieved a favorable anti-tumor effect (response rate: 57\%, PFS: 9.9 months) and caused low incidence of adverse events in frail patients aged 65 years or older in a phase II study (BASIC study), but the number of studies is still insufficient [17]. The efficacy and safety of chemotherapy combined bevacizumab with S-1 or $5 \mathrm{FU} / \mathrm{LV}$ as a first-line for frail patients with unresectable or metastatic colorectal cancer were investigated based on the outcomes obtained at our hospital.

\section{Subjects and Methods}

Subjects

Twenty-six patients diagnosed with unresectable or metastatic colorectal cancer at our hospital between October 2007 and December 2010 in whom treatment was started as chemotherapy combined bevacizumab with S-1 or 5FU/LV [modified Roswell Park Memorial Institute (RPMI) regimen] were retrospectively investigated. They were aged 65 years or older with an Eastern Cooperative Oncology Group (ECOG) performance status of $0-2$ and met one or more of the following conditions: serum albumin $<3.5 \mathrm{~g} / \mathrm{dl}$, past medical history of radiation of the abdominal region or pelvic cavity, and incompatibility with oxaliplatin and irinotecan. This is the same as the eligibility criteria of the AVF2192g study [15].

\section{Treatment Protocol}

In chemotherapy combined bevacizumab with $S-1$, the daily dose of S-1 was set as follows based on the body surface area (BSA): $80 \mathrm{mg} /$ day for BSA $<1.25 \mathrm{~m}^{2}, 100 \mathrm{mg}$ /day for $1.25 \mathrm{~m}^{2} \leq \mathrm{BSA}<$
60

Digestion 2013;87:59-64
Yoshida/Goto/Kii/Nishitani/Kawabe/ Kuwakado/Asaishi/Miyamoto/Higuchi 


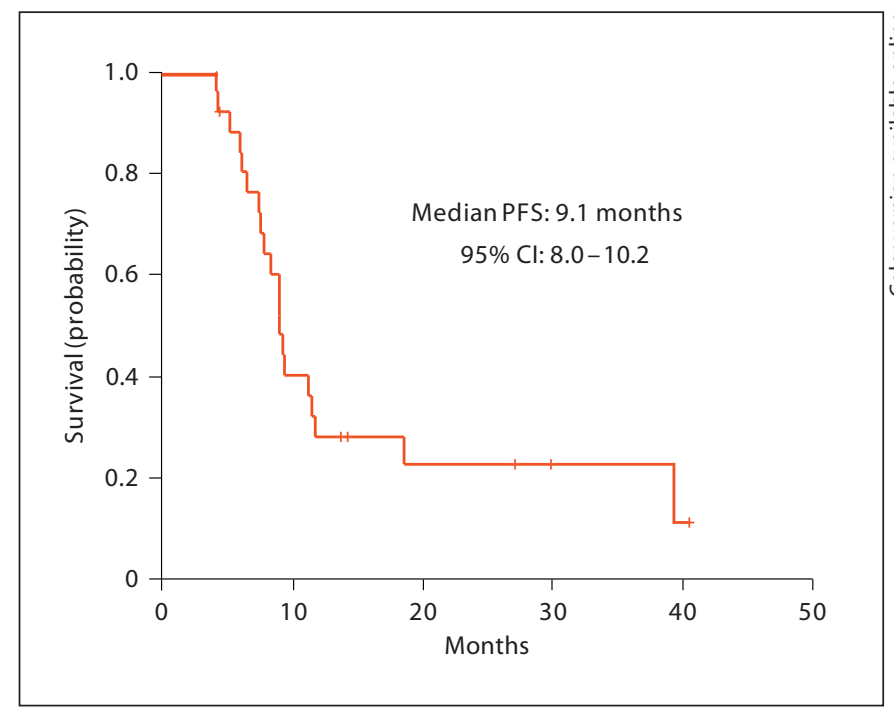

Fig. 1. Progression-free survival.

$1.5 \mathrm{~m}^{2}$, and $120 \mathrm{mg} /$ day for BSA $\geq 1.5 \mathrm{~m}^{2}$. The drug was orally administered twice a day daily for 28 days, followed by a 14-day withdrawal. Bevacizumab was administered at $5 \mathrm{mg} / \mathrm{kg}$ by $30-$ to 90-min drip infusion on days 1, 15 and 29 (2-week intervals). One complete cycle was comprised of a total of 42 days.

In chemotherapy combined bevacizumab with $5 \mathrm{FU} / \mathrm{LV}$ (modified RPMI regimen), $250 \mathrm{mg} / \mathrm{m}^{2}$ of $\mathrm{LV}$ was administered by 2-hour drip infusion and $600 \mathrm{mg} / \mathrm{m}^{2}$ of $5 \mathrm{FU}$ was administered by intravenous bolus injection on days 1,8 and 15 . Bevacizumab was administered at $5 \mathrm{mg} / \mathrm{kg}$ by 30 - to 90 -min drip infusion on days 1 and 15 (2-week interval). One complete cycle was comprised of 28 days.

\section{Assessment of Outcome}

Reponses were evaluated following the Response Evaluation Criteria in Solid Tumors version 1.0 before and after therapy in each patient. Complete response (CR) and partial response (PR) were confirmed when judged twice or more consecutively at a 4 -week or longer interval.

PFS was defined as the time between the days on which consent for treatment initiation was obtained and disease progression was confirmed. When disease progression could not be confirmed, it was defined as the time until death. OS was defined as the time between the treatment initiation and death and, when death could not be confirmed, it was defined as the time until the final confirmation of survival. PFS and OS were analyzed employing the Kaplan-Meier method.

Adverse events were evaluated based on the worst grade observed during the treatment period following the Common Terminology Criteria for Adverse Events (CTCAE) v3.0. Protocol of this retrospective research approval was obtained from an institutional ethics committee at our hospital.

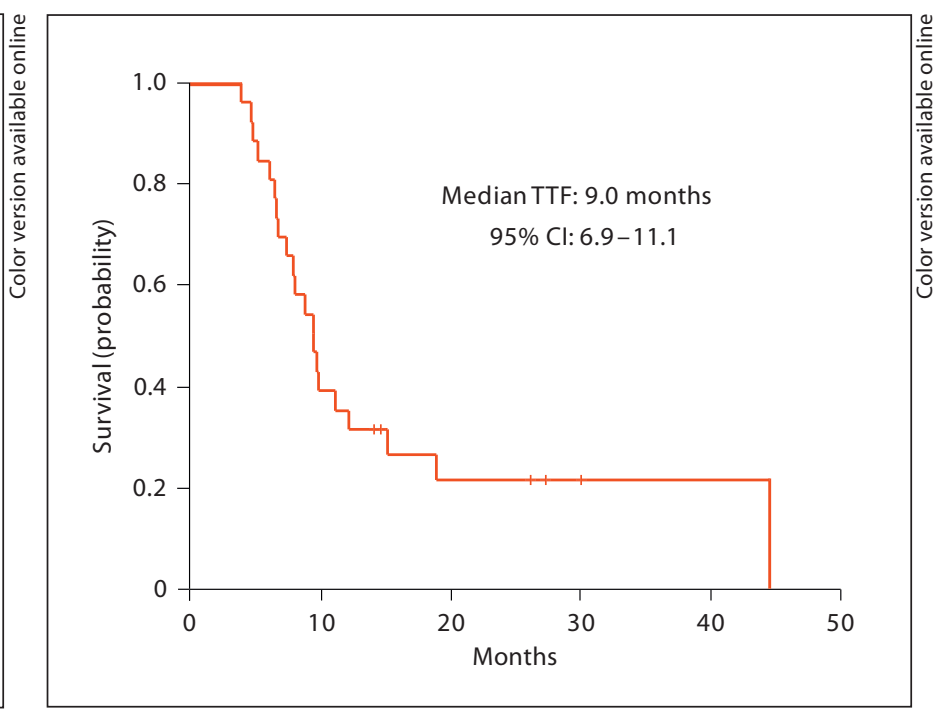

Fig. 2. Time to treatment failure.

Table 1. Patient characteristics $(n=26)$

$\begin{array}{lc}\text { Median age (range) } & 72(66-84) \\ \text { Gender (male/female) } & 9 / 17 \\ \text { ECOG performance status (0/1/2) } & 8 / 17 / 1 \\ \text { Primary site (colon/rectum) } & 14 / 12 \\ \text { Resection (yes/no) } & 20 / 6 \\ \text { Adjuvant chemotherapy (yes/no) } & 8 / 18 \\ \text { Number of metastatic site (1/2/3) } & 17 / 9 / 0 \\ \text { Metastasis } & \\ \quad \text { Liver } & 9 \\ \quad \text { Lung } & 9 \\ \quad \text { Lymph node } & 11 \\ \quad \text { Peritoneum } & 5 \\ \text { KRAS gene (wild/mutated/unknown) } & 11 / 7 / 8 \\ \text { S-1 + bevacizumab/5FU + bevacizumab } & 17 / 9\end{array}$

\section{Results}

The patient characteristics are shown in table 1. Median age was 72 years (range 66-84). ECOG performance status was 0,1 and 2 in 8,17 and 1 patient, respectively. The primary lesion was located in the colon in 14 patients and in the rectum in 12 . Twenty patients were with resection of the primary lesion and 6 were without, 8 were with postoperative adjuvant chemotherapy and 18 were without. The number of metastasized organs was 1,2 and 3 in 17, 9 and 0 patients, respectively. The liver, lung, lymph node and peritoneum was metastasized in 9, 9, 11 and 5 patients, 
Table 2. Adverse Events by CTCAE v3.0

\begin{tabular}{|c|c|c|c|c|c|c|c|}
\hline \multirow[t]{2}{*}{ Event } & \multicolumn{5}{|c|}{ Grade } & \multirow{2}{*}{$\begin{array}{l}\text { Any } \\
\text { grade } \\
\%\end{array}$} & \multirow{2}{*}{$\begin{array}{l}\text { Grade } \\
>3 \\
\%\end{array}$} \\
\hline & 1 & 2 & 3 & 4 & 5 & & \\
\hline Neutropenia & 1 & 5 & 2 & 0 & 1 & 31 & 12 \\
\hline Thrombocytopenia & 8 & 3 & 0 & 0 & 0 & 42 & 0 \\
\hline Anemia & 14 & 5 & 1 & 0 & 0 & 77 & 4 \\
\hline Nausea & 9 & 0 & 0 & 0 & 0 & 35 & 0 \\
\hline Diarrhea & 6 & 2 & 1 & 0 & 0 & 35 & 4 \\
\hline Fatigue & 13 & 0 & 0 & 0 & 0 & 50 & 0 \\
\hline Stomatitis & 8 & 0 & 0 & 0 & 0 & 31 & 0 \\
\hline Hand-foot syndrome & 10 & 2 & 0 & 0 & 0 & 46 & 0 \\
\hline Hypertension & 3 & 2 & 3 & 0 & 0 & 31 & 12 \\
\hline Proteinuria & 4 & 11 & 0 & 0 & 0 & 58 & 0 \\
\hline Epistaxis & 9 & 0 & 0 & 0 & 0 & 35 & 0 \\
\hline Cerebral hemorrhage & 0 & 0 & 1 & 0 & 0 & 4 & 4 \\
\hline Thrombosis & 0 & 0 & 0 & 1 & 0 & 4 & 4 \\
\hline
\end{tabular}

respectively. The KRAS gene was wild in 11, mutant in 7 and unknown in 8 patients, and treatment was chemotherapy combined bevacizumab with S-1 in 17 patients and chemotherapy combined bevacizumab with 5FU/LV in 9.

Regarding the efficacy, response was CR in 1, PR in 12, stable disease in 13, progressive disease in 0 and not evaluated 0 patients. The response rate was $50 \%$ and disease control rate was $100 \%$. The median PFS was 9.1 months (95\% CI 8.0-10.2; fig. 1). The median time to treatment failure was 9.0 months (95\% CI 6.9-11.1; fig. 2), median OS was 28.9 months (95\% CI 19.6-38.2) and median duration of follow-up was 20.7 months.

The mean relative dose intensity of chemotherapy combined bevacizumab with S-1 was: S-1 94.0\% (range 66.5-100) and bevacizumab 62.0\% (range 3.1-100), and that of with 5FU/LV was: 5FU 76.6\% (range 68.6-91.3) and bevacizumab 71.7\% (range 49.0-91.3).

The incidence of neutropenia at all grades was $31 \%$ and that of grade 3 or severer was $12 \%$, the incidence of hypertension at all grades was also $31 \%$ and that of grade 3 or severer was $12 \%$, showing low incidence, and those of other adverse events were also generally low. Grade 3 cerebral hemorrhage, grade 4 pulmonary embolus and grade 5 febrile neutropenia occurred each in 1 patient (table 2). First-line therapy was continued in 4 patients (15\%), discontinuation of therapy occurred due to the following reasons: CR in 1 patient (4\%), resection of liver metastasis in $1(4 \%)$ or lung metastasis in $1(4 \%)$, disease progression in $18(69 \%)$ and adverse events in $3(12 \%$; cerebral hemorrhage, pulmonary embolus and FN). Excluding 7 patients under the first-line of therapy or course observation, $84 \%$ of patients received second-line chemotherapy: FOLFOX/XELOX was administered to 5 patients, FOLFIRI/IRI to 9 and 5FU/LV to 2 .

\section{Discussion}

We investigated the safety and efficacy of chemotherapy combined bevacizumab with fluoropyrimidine for elderly or frail patients with unresectable or metastatic colorectal cancer. The response rate was $50 \%$, disease control rate was $100 \%$ and PFS was 9.1 months. These result suggest that such combined therapy might be useful for frail patients. However, this was a retrospective study performed at a single facility, not a prospective clinical study, and no statistical endpoint was established, showing the limitations of the study. Moreover, patient selection was biased and the OS observation period was short, indicating that the data are still immature.

Several clinical studies have reported the efficacy and safety of chemotherapy combined bevacizumab with fluoropyrimidine. In the AVF2192g randomized controlled phase II study involving frail patients, the primary endpoint, PFS, was 5.5 months in 5FU/LV therapy and 9.2 months in chemotherapy combined bevacizumab with $5 \mathrm{FU} / \mathrm{LV}$, suggesting the usefulness of combination with bevacizumab, and the therapy was tolerated with regard to safety [15]. Similarly, in the MAX study, which was a phase III study of chemotherapy combined bevacizumab with capecitabine, PFS was 5.7 months in capecitabine monotherapy and 8.5 months in chemotherapy combined bevacizumab with capecitabine, showing the usefulness of combination with bevacizumab [16]. In a phase II study of chemotherapy combined bevacizumab with capecitabine involving elderly patients, PFS was 10.8 months, suggesting high-level efficacy, and tolerability was also high [18]. In Japan, marked efficacy was also obtained in a phase II study (BASIC study) of chemotherapy combined bevacizumab with S-1 involving 65-year-old or older frail patients, in which PR was 57\% and PFS was 9.9 months [17].

Regarding the efficacy, the common points of reports on chemotherapy combined bevacizumab with fluoropyrimidine are a slightly lower response rate than that of the standard combination therapy but comparable disease control rates and PFS. Based on these common findings, the standard combination therapy may be desirable for patients requiring tumor size reduction at the time of treatment initiation, but chemotherapy combined bevacizumab with fluoropyrimidine may be appropriate for patients for whom marked tumor size reduction is not nec- 
essary but stabilization of the disease condition is, i.e. cases aiming at curative resection after tumor size reduction and treatment for those with no tumor-associated symptoms. It has been reported that the efficacy of combination therapy with oxaliplatin or irinotecan does not differ between elderly and young patients [9-14]. The efficacy of chemotherapy combined bevacizumab with fluoropyrimidine may also be similar in elderly and young patients because PFS in chemotherapy combined bevacizumab with capecitabine involving elderly patients in a phase II study was not markedly different from that in the MAX study, in which young patients were included $[16,18]$.

Regarding safety, the common points of reports on chemotherapy combined bevacizumab with fluoropyrimidine were a lower incidence of adverse events, particularly neutropenia, than that in clinical studies of the standard combination therapy, and the incidences of all grades and grade 3 or severer neutropenia were 31 and $12 \%$, respectively [1-3]. The incidence of bone marrow toxicity, such as neutropenia, is high in combination therapies with oxaliplatin and irinotecan, particularly in elderly patients [11-16], and the induction of febrile neutropenia is of concern.

Many colorectal cancer patients are elderly, aged 65 years or older, and complications increase as patient age advances [19], meaning it can be difficult to apply chemotherapy to frail patients due to the increasing likelihood of complications. Since the patients in this study were also 65 years old or older, it included many frail patients. Regarding adverse events, the incidences of all grades and grade 3 or severer hypertension were 31 and $12 \%$, respectively, which were higher than those in reported clinical studies. This may have been due the high age of the patients, rather than blood pressure elevation newly induced by bevacizumab.

One patient under chemotherapy combined bevacizumab with S-1 developed proteinuria in the 2nd cycle and bevacizumab administration was discontinued after day 15 in that cycle. Accordingly, the relative dose intensity of bevacizumab was 3\% in this patient, but dose intensity of bevacizumab in chemotherapy combined bevacizumab with S-1 was high (81\%) in the other 16 patients, and proteinuria in this patient was reversible. Therefore, the tolerability of the therapies was high, suggesting highlevel safety. However, grade 3 cerebral hemorrhage, grade 4 pulmonary embolism and grade 5 febrile neutropenia occurred each in 1 patient. Performance status is likely to decrease earlier in elderly or frail patients than in young patients as adverse events develop, requiring treatment as early as possible in many cases. Thus, attention should be paid to the development of adverse events. In actual clinical practice, standard combination chemotherapy is started at a dose lower than the recommended dose because of safety concerns in relatively many cases, but the efficacy of chemotherapy administered at a reduced dose has not been clarified. It is possible that the efficacy of sequential chemotherapy without dose reduction is higher than standard combination chemotherapy with dose reduction.

Although there have been fewer reports of clinical studies on the usefulness of sequential chemotherapy using molecular target drugs, there was no significant difference in OS between sequential chemotherapy started with fluoropyrimidine monotherapy as the first-line treatment followed by oxaliplatin or irinotecan as the second-line or later treatment, and chemotherapy combined fluoropyrimidine with oxaliplatin or irinotecan as the first-line therapy in the FOCUS [4], CAIRO [5] and FFCD 2000-2005 studies [6], suggesting the usefulness of sequential chemotherapy.

In a study of chemotherapy combined fluoropyrimidine with anti-EGFR antibody involving untreated elderly patients, the response rate was $31.8 \%$ (95\% CI $20.9-$ 44.4) and PFS was 7.1 months, which is not superior to treatment with anti-EGFR antibody preparation alone, and the incidences of grade 3 or severer adverse events were high (skin toxicity $30 \%$, hand-foot syndrome $22 \%$ and diarrhea $18.5 \%$ ), showing that it is not a favorable relationship [20].

In conclusion, the first-line chemotherapy combined bevacizumab with fluoropyrimidine for unresectable or metastatic colorectal cancer frail patients in Japan was comparable to the safety and efficacy of combination therapy reported previously in Western countries. Because chemotherapy combined anti-VEGF antibody with fluoropyrimidine is lower than the other chemotherapy regimen regarding toxicity, further clinical studies may be necessary for such combined chemotherapy not only as a treatment more tolerable for frail patients than other drug therapies, but also as the first-line treatment of sequential chemotherapy.

\section{Disclosure Statement}

The author received small payments for lectures from Chugai Pharmaceutical Co. Ltd. and Taiho Pharmaceutical Co. Ltd. Grants were also provided to the Chemotherapy Center by these 2 pharmaceutical companies. 


\section{References}

1 Saltz LB, Clarke S, Diaz-Rubio E, Scheithauer W, Figer A, Wong R, Koski S, Lichinitser M, Yang TS, Rivera F, Couture F, Sirzén F, Cassidy J: Bevacizumab in combination with oxaliplatin-based chemotherapy as first-line therapy in metastatic colorectal cancer: a randomized phase III study. J Clin Oncol 2008;26:2013-2019.

$>2$ Cutsem EV, Köhne CH, Hitre E, Zaluski J, Chang Chien CR, Makhson A, D’Haens G, Pintér T, Lim R, Bodoky G, Roh JK, Folprecht G, Ruff P, Stroh C, Tejpar S, Schlichting M, Nippgen J, Rougier P: Cetuximab and chemotherapy as initial treatment for metastatic colorectal cancer. N Engl J Med 2009;360: 1408-1417.

$>3$ Douillard JY, Siena S, Cassidy J, Tabernero J, Burkes R, Barugel M, Humblet Y, Bodoky G, Cunningham D, Jassem J, Rivera F, Kocákova I, Ruff P, Błasińska-Morawiec M, Šmakal M, Canon JL, Rother M, Oliner KS, Wolf M, Gansert J: Randomized, phase III trial of panitumumab with infusional fluorouracil, leucovorin, and oxaliplatin (FOLFOX4) versus FOLFOX4 alone as first-line treatment in patients with previously untreated metastatic colorectal cancer: the PRIME study. J Clin Oncol 2010;28:4697-4705.

4 Seymour MT, Maughan TS, Ledermann JA, Topham C, James R, Gwyther SJ, Smith DB, Shepherd S, Maraveyas A, Ferry DR, Meade AM, Thompson L, Griffiths GO, Parmar MK, Stephens RJ, et al: Different strategies of sequential and combination chemotherapy for patients with poor prognosis advanced colorectal cancer (MRC FOCUS): a randomised controlled trial. Lancet 2007;370: 143-152.

5 Koopman M, Antonini NF, Douma J, Wals J, Honkoop AH, Erdkamp FL, de Jong RS, Rodenburg CJ, Vreugdenhil G, Loosveld OJ, van Bochove A, Sinnige HA, Creemers GJ, Tesselaar ME, Slee PH, Werter MJ, Mol L, Dalesio O, Punt CJ: Sequential versus combination chemotherapy with capecitabine, irinotecan and oxaliplatin in advanced colorectal cancer (CAIRO): a phase III randomized controlled trial. Lancet 2007;370:135-142.
6 Ducreux M, Malka D, Mendiboure J, Etienne PL, Texereau P, Auby D, Rougier P, Gasmi M, Castaing M, Abbas M, Michel P, Gargot D, Azzedine A, Lombard-Bohas C, Geoffroy P, Denis B, Pignon JP, Bedenne L, Bouché O, Fédération Francophone de Cancérologie Digestive (FFCD) 2000-05 Collaborative Group: Sequential versus combination chemotherapy for the treatment of advanced colorectal cancer (FFCD 2000-05): an openlabel, randomised, phase 3 trial. Lancet Oncol 2011;12:1032-1044.

7 Adam R, Wicherts DA, de Haas RJ, Ciacio O, Lévi F, Paule B, Ducreux M, Azoulay D, Bismuth $\mathrm{H}$, Castaing D: Patients with initially unresectable colorectal liver metastases: is there a possibility of cure? J Clin Oncol 2009; 27:1829-1835.

-8 Cutsem EV, Nordlinger B, Cervantes A, ESMO Guidelines Working Group: Advanced colorectal cancer: ESMO clinical practice guidelines for treatment. Ann Oncol 2010;21(suppl 5):v93-v97.

$\checkmark 9$ Figer A, Perez-Staub N, Carola E, Tournigand C, Lledo G, Flesch M, Barcelo R, Cervantes A, André T, Colin P, Louvet C, de Gramont A: FOLFOX in patients aged between 76 and 80 years with metastatic colorectal cancer: an exploratory cohort of the OPTIMOX1 study. Cancer 2007;110:2666-2671.

10 Tournigand C, Cervantes A, Figer A, Lledo G, Flesch M, Buyse M, Mineur L, Carola E, Etienne PL, Rivera F, Chirivella I, PerezStaub N, Louvet C, André T, Tabah-Fisch I, de Gramont A: A randomized study of FOLFOX4 or FOLFOX7 with oxaliplatin in a stop-and-go fashion in advanced colorectal cancer - a GERCOR study. J Clin Oncol 2006;24:394-400.

11 Jackson NA, Barrueco J, Soufi-Mahjoubi R, Marshall J, Mitchell E, Zhang X, Meyerhardt $\mathrm{J}$ : Comparing safety and efficacy of first-line irinotecan/fluoropyrimidine combinations in elderly versus nonelderly patients with metastatic colorectal cancer. Cancer 2009; 115:2617-2629.

12 Hurwitz H, Fehrenbacher L, Novotny W, Cartwright T, Hainsworth J, Heim W, Berlin J, Baron A, Griffing S, Holmgren E, Ferrara N, Fyfe G, Rogers B, Ross R, Kabbinavar F: Bevacizumab plus irinotecan, fluorouracil, and leucovorin for metastatic colorectal cancer. N Engl J Med 2004;350:2335-2342.

-13 Kabbinavar FF, Hambleton J, Mass RD, Hurwitz HI, Bergsland E, Sarkar S: Combined analysis of efficacy: the addition of Bevacizumab to fluorouracil/leucovorin improves survival for patients with metastatic colorectal cancer. J Clin Oncol 2005;23:3706-3712.
14 Saltz LB, Clarke S, Díaz-Rubio E, Scheithauer W, Figer A, Wong R, Koski S, Lichinitser M, Yang TS, Rivera F, Couture F, Sirzén F, Cassidy J: Bevacizumab in combination with oxaliplatin-based chemotherapy as first-line therapy in metastatic colorectal cancer: a randomized phase III study. J Clin Oncol 2008;26:2013-2019.

15 Kabbinavar FF, Schulz J, McCleod M, Patel T, Hamm JT, Hecht JR, Mass R, Perrou B, Nelson B, Novotny WF: Addition of bevacizumab to bolus fluorouracil and leucovorin in first-line metastatic colorectal cancer: results of a randomized phase II trial. J Clin Oncol 2005;23:3697-3705.

16 Tebbutt NC, Wilson K, Gebski VJ, Cummins MM, Zannino D, van Hazel GA, Robinson B, Broad A, Ganju V, Ackland SP, Forgeson G, Cunningham D, Saunders MP, Stockler MR, Chua Y, Zalcberg JR, Simes RJ, Price TJ: Capecitabine, bevacizumab and mitomycin in first-line treatment of metastatic colorectal cancer: results of the Australasian Gastrointestinal Trials Group Randomized Phase III MAX study. J Clin Oncol 2010;28: 3191-3198.

17 Takahari D, Takiuchi H, Muro K, Tsuji A, Hamamoto Y, Yoshino T, Yoshida K, Shirao K, Miyata Y, Ohtsu A: Phase II trial of combination therapy with bevacizumab and S-1 in elderly patients with unresectable or recurrent colorectal cancer (BASIC). Eur J Cancer Abstract Book 2011(abstr 6121):428429.

18 Feliu J, Safont MJ, Salud A, Losa F, GarcíaGirón C, Bosch C, Escudero P, López R, Madroñal C, Bolaños M, Gil M, Llombart A, Castro-Carpeño J, González-Barón M: Capecitabine and bevacizumab as first-line treatment in elderly patients with metastatic colorectal cancer. Br J Cancer 2010;102: 1468-1473.

19 Yancik R, Wesley MN, Ries LA, Havlik RJ, Long S, Edwards BK, Yates JW: Comorbidity and age as predictors of risk for early mortality of male and female colon carcinoma patients: population-based study. Cancer 1998; 82:2123-2134.

20 Sastre J, Grávalos C, Rivera F, Massuti B, Valladares-Ayerbes M, Marcuello E, Manzano JL, Benavides M, Hidalgo M, Díaz-Rubio E, Aranda E: First-line cetuximab plus capecitabine in elderly patients with advanced colorectal cancer: clinical outcome and subgroup analysis according to KRAS status from a Spanish TTD group study. Oncologist 2012;17:339-345. 